

\title{
APROXIMACIÓN AL OBJETO DE ESTUDIO DE LA EDUCACIÓN FÍSICA MILITAR DESDE LAS CONCEPCIONES DE EDUCACIÓN FÍSICA Y CUERPO ${ }^{1}$
}

\author{
AN APPROACH TO THE OBJECT OF STUDY OF MILITARY PHYSICAL EDUCATION BASED ON \\ THE CONCEPTIONS OF PHYSICAL EDUCATION AND THE BODY
}

\section{APROXIMAÇÃO AO OBJETIVO DE ESTUDO DA EDUCAÇÃO FÍSICA MILITAR DESDE AS CONCEPÇÕES DE EDUCAÇÃO FÍSICA E CORPO}

\author{
Osman Díaz-Herrera ${ }^{2}$ \\ Paula Melo-Buitrago ${ }^{3}$ \\ Santiago Cortés-Fernandez ${ }^{4}$
}

Resumen

La Educación Física Militar en Colombia se imparte en la Facultad de Educación Física de la Escuela Militar de Cadetes General José María Córdova, la cual cuenta con un programa profesional que busca establecer con mayor claridad su objeto de estudio, conforme a la estructuración, en primera medida, de las concepciones de educación física y cuerpo. Este artículo es producto de una investigación que tuvo como objetivo aproximarse al objeto de estudio de la educación física militar desde estas concepciones. Se siguió una metodología etnográfica y se contó con la participación de cuatro (4) docentes del programa Profesional en Educación Física Militar de la Escuela Militar de Cadetes, así como con cuatro (4) docentes del Proyecto Curricular Licenciatura en Educación Física de la Universidad Pedagógica Nacional. Como resultado se establecieron tres (3) categorías deductivas y emergieron diez y seis (16) categorías inductivas, las cuales permitieron concluir que la marcada predominancia de hábitos e ideas castrenses en la cotidianidad del contexto militar promueve una educación física y cuerpos normativizados en busca de la eficiencia bélica y el rendimiento físico.

Palabras clave: objeto de estudio; paradigma; educación física; educación física militar; cuerpo

\footnotetext{
1 Artículo de investigación, resultado de la investigación "Configuración de paradigmas propios que fundamenten el objeto de estudio de la Educación Física Militar", llevado a cabo en la Escuela Militar de Cadetes General José María Córdova y la Universidad Pedagógica Nacional durante el periodo 2015-2016, desarrollado en el grupo de investigación Fortius, en la línea de Epistemología de la Educación Física Militar de la Facultad de Educación Física Militar de la Escuela Militar de Cadetes.

2 Docente de la Facultad de Educación Física Militar, Escuela Militar de Cadetes General José María Córdova. Entrenador de Fútbol Base de Millonarios F. C. Magíster en Ciencias y Tecnologías del Deporte y la Actividad Física por la Universidad Manuela Beltrán. Especialista en Preparación Física en el Fútbol por la Universidad Politécnico Jaime Isaza Cadavid. Especialista en Pedagogía y Didáctica de la Educación Física por la Universidad Pedagógica Nacional. Profesional en Ciencias del Deporte y de la Educación Física por la Universidad de Cundinamarca. Correo electrónico: osmandiazherrera@hotmail.com.

3 Docente de la Facultad de Educación Física Militar y coordinadora de investigaciones de la Escuela Militar de Cadetes General José María Córdova, líder del grupo de investigación Fortius de la línea de investigación Entrenamiento Físico Militar. Licenciada en Educación Física, Recreación y Deporte por la Universidad de Cundinamarca. Magíster en Educación con Énfasis en Fisiología del Deporte por la Universidad del Valle. Coordinadora de investigaciones de la Facultad de Educación Física Militar de la Escuela Militar de Cadetes General José María Córdova. Correo electrónico: paulajanynmb@yahoo.es.

4 Decano de la Facultad de Educación Militar de la Escuela Militar de Cadetes General José María Córdova. oficial del Ejército Nacional (capitán). Magíster en Ciencias y Tecnologías del Deporte y la Actividad Física por la Universidad Manuela Beltrán. Especialista en Altos Estudios del Deporte por la Universidad Jorge Tadeo Lozano. Licenciado en Educación Física, Recreación y Deporte de la Universidad Pedagógica Nacional. Correo electrónico: sacofe@hotmail.com.
} 


\section{Abstract}

Military physical education in Colombia is taught at the School of Physical Education of the Escuela Militar de Cadetes General José María Córdova, which offers a professional program that seeks to establish its object of study more clearly. This article is the product of a research that aimed to approach the object of study of military physical education based on the conceptions of physical education and body. An ethnographic methodology was used, with the participation of four (4) teachers from the Professional Program in Military Physical Education of the Escuela Militar de Cadetes, as well as four (4) teachers from the Curricular Project from the Bachelor in Physical Education of Universidad Pedagógica Nacional. As a result, three (3) deductive categories were established, and sixteen (16) inductive categories emerged, which made it possible to conclude that the marked predominance of military habits and ideas in the daily life of the military context promotes normative physical education and bodies, in the search for military efficiency and physical performance.

Keywords: object of study; paradigm; physical education; military physical education; body

\section{Resumo}

A Educação Física Militar na Colômbia é ministrada na Faculdade de Educação Física da Escola Militar de Cadetes Geral José Maria Córdova. Existe um programa profissional que procura estabelecer com claridade seu objetivo de estudo, conforme a estruturação, em primeiro lugar, das concepções da educação física e corpo. Este artigo é produto de uma pesquisa que teve por objetivo abordar o objeto de estudo da educação física militar desde essas concepções. Sugeriu-se uma metodologia etnográfica para a pesquisa, na qual participaram quatro (4) professores do programa profissional em Educação Física Militar da Escola Militar de Cadetes, assim como quatro (4) professores do Projeto Curricular Licenciatura em Educação Física da Universidade Pedagógica Nacional. Como resultado, foram estabelecidas três (3) categorias dedutivas e emergiram dezesseis (16) categorias indutivas, que permitiram concluir que a predominância de hábitos e ideias castrenses no dia-a-dia do contexto militar promove uma educação física e corpos normatizados na procura da eficiência bélica e do rendimento físico.

Palavras chave: objeto de estudo; paradigma; educação física; educação física militar; corpo

Fecha de recepción: 7 de mayo de 2016

Fecha de aprobación: 20 de octubre de 2016

Para citar este artículo:

Cortés, S., Díaz, O. y Melo, P. (2017). Aproximación al objeto de estudio de la educación física militar desde las concepciones de educación física y cuerpo. Lúdica Pedagógica, (25), 95-104. 


\section{INTRODUCCIÓN}

La Educación Física Militar en Colombia se imparte en la Facultad de Educación Física de la Escuela Militar de Cadetes, la cual cuenta con un programa profesional en premura de establecer con mayor claridad su objeto de estudio (Centro Nacional de Acreditación CNA, 2012). El análisis e interpretación de las categorías de educación física y cuerpo a partir de las concepciones de los expertos es un punto de partida determinante para configurar el objeto de estudio que, posteriormente -más allá de que la necesidad parta de un requerimiento administrativo-, posibilite estructurar el cuerpo teórico, el enfoque disciplinar específico, las características didácticas, los componentes del currículo y los procesos investigativos. Estos influirán directamente en la cualificación de las prácticas educativas y, por ende, en la transformación de los sujetos a partir de los principios y valores del Ejército Nacional (Ejército Nacional de Colombia, 2016).

Sin embargo, la necesidad de configurar un objeto de estudio claro y soportado desde las diferentes corrientes epistemológicas y paradigmas de la educación física y el cuerpo es una pretensión compleja debido a todos los elementos que se entrecruzan en su construcción. Estos cruces sugieren una interpretación y análisis que lleven a indagar sobre los ejes específicos que atraviesan las categorías de educación física y cuerpo. En consecuencia, conviene preguntarse si las concepciones de educación física y cuerpo permiten una aproximación al objeto de estudio de la educación física militar, ya que, precisamente, algunos paradigmas de educación física sustentan su objeto de estudio en el cuerpo (Ministerio de Educación Nacional [MEN], 2000).

\section{OBJETIVO}

Realizar una aproximación al objeto de estudio de la educación física militar desde las concepciones de educación física y cuerpo.

\section{MÉTODO}

Se utilizó el enfoque investigativo cualitativo, al cual le interesan los datos por sus cualidades, por lo que ocurre en cada uno de ellos, no tanto por sus cantidades. Su fundamento está dado por la visión holística de las realidades, lo cual implica comprenderlas desde la relación que hay entre cada uno de sus elementos y no desde la visión aislada de ellos (Cerda, 1995; Rodríguez, 2011).

Se adoptó una metodología etnográfica, por considerar que posibilita el descubrimiento, la reconstrucción analítica y el aporte de valiosos datos descriptivos acerca de las concepciones de los docentes tanto del programa Profesional en Educación Física Militar de la Escuela Militar de Cadetes como del Proyecto Curricular Licenciatura en Educación Física de la Universidad Pedagógica Nacional.

\section{Fundamentación teórica de}

la metodología etnográfica

Partiendo de su etimología, etnografía significa la descripción (grafé) del estilo de vida de un grupo de personas habituadas a vivir juntas (ethnos). Por lo tanto, el ethnos que sería la unidad de análisis para el investigador no solo podría ser una nación, un grupo lingüístico, una región o una comunidad, sino también cualquier grupo humano que constituya una entidad, cuyas relaciones estén reguladas por la costumbre o por ciertos derechos y obligaciones recíprocos (Rodríguez, 2011).

Un producto etnográfico se evalúa por la medida en que logra una recreación del escenario cultural estudiado, que permita a los lectores representárselo tal como apareció ante la mirada del investigador. Además de producto, la etnografía es un proceso, una forma de estudiar la vida humana.

El diseño etnográfico requiere estrategias de investigación que conduzcan a la reconstrucción cultural. En principio, estas proporcionan datos fenomenológicos que representan la concepción del mundo de los participantes que están siendo investigados, de forma que sus constructos se utilicen para estructurar la investigación (Camargo, 1995; De Tezanos, 1998).

Además, las estrategias etnográficas de investigación son empíricas y naturalistas. Se obtienen datos empíricos de primera mano de los fenómenos tal como se dan en el mundo real, en tanto que los investigadores procuran evitar la manipulación intencional de las categorías de estudio. Por último, la investigación etnográfica tiene carácter holista. Pretende construir descripciones de sus fenómenos globales en sus diversos contextos y 
determinar, a partir de ellas, las complejas conexiones de causas y consecuencias que afectan el comportamiento y las creencias en relación con dichos fenómenos.

\section{Población}

El presente estudio se realizó con cuatro docentes del programa Profesional en Educación Física Militar de la Escuela Militar de Cadetes (registros 1, 2, 3 y 4) y cuatro del Proyecto Curricular Licenciatura en Educación Física de la Universidad Pedagógica Nacional (registros: 5, 6, 7 u 8). Se utilizó una muestra no probabilística, pues los datos analizados no se refieren a parámetros poblacionales sino que se busca la selección de casos (individuos) que cumplan con las características requeridas por la investigación. Al respecto, se recurrió a una muestra de expertos, ya que su objetivo "es la riqueza, profundidad y calidad de la información, no la cantidad ni la estandarización" (Hernández-Sampieri, Fernández-Collado y Baptista-Lucio, 2010, p. 397). Los criterios de inclusión fueron: llevar más de cinco años como docente en el programa y tener, como mínimo, estudios de maestría.

\section{Instrumento}

Se utilizó un cuestionario de preguntas abiertas que consta de dos ítems, orientadas a partir de las categorías deductivas que se preestablecieron para el estudio. Las preguntas son las mismas para los docentes de cada programa, sin embargo, cuentan con la contextualización correspondiente según el caso (tabla 1).

Tabla 1. Preguntas y categorías deductivas del cuestionario

\begin{tabular}{|l|l|}
\hline \multicolumn{1}{|c|}{ Preguntas } & \multicolumn{1}{|c|}{ Categorías deductivas } \\
\hline $\begin{array}{l}\text { ¿Cuál es su concepto acerca } \\
\text { de la educación física militar? }\end{array}$ & $\begin{array}{l}\text { Concepción de educación } \\
\text { física militar }\end{array}$ \\
\hline $\begin{array}{l}\text { ¿Cuál es su concepto acerca de } \\
\text { la educación física? }\end{array}$ & $\begin{array}{l}\text { Concepción de educación } \\
\text { física }\end{array}$ \\
\hline $\begin{array}{l}\text { En el contexto de la educación } \\
\text { física militar, ¿qué concepto de } \\
\text { cuerpo se establece? }\end{array}$ & \\
\hline $\begin{array}{l}\text { En el contexto de la educa- } \\
\text { ción física, ¿qué concepto de } \\
\text { cuerpo se establece? }\end{array}$ & Concepción de cuerpo \\
\hline
\end{tabular}

Fuente: elaboración propia

Técnicas de procesamiento y análisis de datos

Para el procesamiento y análisis de los datos se utilizó una matriz de registros que posibilitó la organización y categorización de la información con el fin de clasificar las partes en relación con el todo, de describir categorías o clases significativas, de ir constantemente diseñando y rediseñando, integrando y reintegrando el todo y las partes. A medida que se revisaba el material, fue emergiendo el significado de cada sector, evento, dato o hecho -categorías inductivas (Bonilla y Rodríguez, 1997). La matriz contiene tanto categorías deductivas como inductivas (Hernández-Sampieri et al., 2010) y se presentará en los resultados de este artículo.

\section{MARCO TEÓRICO}

Reseña histórica de la educación física militar en Colombia

En Colombia, el general Francisco de Paula Santander, durante su presidencia, fue pionero en la implementación de planes de estudio de educación física. Estos servían como mecanismo de preservación de la salud del escolar y dotaban al practicante de hábitos higiénicos que prevenían las enfermedades y lo predisponían a la obtención de un estado óptimo de rendimiento para el desarrollo de sus diversas actividades cotidianas. Posteriormente, a principios del siglo xx, se presentaron las primeras influencias de carácter gimnástico que ya habían tenido éxito en países como Francia, Inglaterra, Alemania y Suecia. De igual manera, las grandes guerras mundiales direccionaron e influenciaron los procesos de formación en este ámbito en Colombia, aportando actividades como formaciones militares, promoción de la disciplina por medio de órdenes y el control en las clases mediante castigos físicos, ya que los profesores solían ser militares que terminaban su servicio y después se vinculaban a instituciones escolares (CNA, 2012).

En las Fuerzas Militares la influencia de la preparación física y de los deportes ha estado asentada en el tiempo. Son famosas las gestas deportivas de los militares (especialmente en los inicios de la participación deportiva nacional), y no tan bien recordados los procesos de entrenamiento físico que se llevaban a cabo, quizás por su exigencia y por el maltrato que se puede evidenciar en los relatos de quienes lo sufrieron (CNA, 2012).

Debido a lo anterior, las posibilidades académicas para los oficiales del Ejército Nacional, que habían tenido etapas de surgimiento y desaparición desde los años 60 -merece la pena mencionar que la Universidad Militar 
Nueva Granada tuvo su origen en la Escuela Militar-, obtuvieron su posicionamiento definitivo en el año 2000 cuando surgió el Plan de Estudios de Educación Física Militar, el primer programa de Educación Física con registro Icfes en funcionar sin titular a sus egresados, con la expectativa de que, con estudios iniciados, los oficiales terminaran y obtuvieran sus títulos profesionales "por su cuenta", a través de procesos de homologación en las diferentes regiones del país. No obstante, la realidad misional y operacional demostró la imposibilidad de esta iniciativa y por ello se ajustó el plan de estudios en procura de la nueva exigencia del men de contar con registros calificados, requisito que se cumplió en junio de 2008. Así nació la Facultad de Educación Física Militar (CNA, 2012) con su Programa de Educación Física Militar, el cual obtuvo la Acreditación de Alta Calidad Educativa en noviembre de 2012.

\section{Consideraciones epistemológicas iniciales}

La configuración de un objeto de estudio, en cualquier disciplina del conocimiento, requiere tener como punto de partida la epistemología, entendida como aquella rama filosófica que estudia el conocimiento científico en un contexto histórico-social determinado, en aras de la búsqueda de la verdad (Bachelard, 1973). El objeto de estudio es el eje transversal de todo plan de estudios de Educación Física y su configuración inicia con las concepciones de cuerpo y educación física (men, 2000). Al respecto, para realizar una aproximación más expedita a lo que es un objeto de estudio, es necesario revisar el concepto de paradigma entendido desde Kuhn (1969), quien lo considera como la interpretación de un fenómeno en un contexto social determinado desde un marco teórico-metodológico. Lo anterior, posibilita expresar que diferentes paradigmas de educación física han orientado su quehacer al objeto de estudio como fenómeno social-teórico-metodológico. Algunos ejemplos de objetos de estudio en la educación física son: el cuerpo y el juego, el hombre en movimiento, educación por el movimiento, el movimiento como acción, cultura del movimiento, deporte, conducta motriz- acción motriz, experiencia corporal (MEN, 2000).

\section{Concepción de educación física}

No se puede desconocer que tanto la educación física en general como la educación física militar en particular han sido permeadas por los diversos cambios ocurridos en los ámbitos social, económico, político y cultural. Por esta razón, a lo largo de la historia de la humanidad se ha evidenciado variedad en sus paradigmas, tendencias, discursos, concepciones, actores y prácticas (Rosa, 1992). Así, la condición física, como tendencia y como paradigma de la enseñanza de la educación física, señala a las escuelas gimnásticas como la primera manifestación sistemática y organizada de la educación física. Además, los discursos religiosos, médicos y educativos sobre la base de una estructura sociopolítica posibilitaron su auge y aprobación tanto social como escolar. Es en la gimnasia donde se encuentran las primeras formas de la educación física escolar (Fernández, 2007). La gimnástica escolar justifica su incursión en el ámbito educativo por ser considerada un medio para lograr el desarrollo integral de los niños, es decir, el objetivo primordial era que su práctica regular incidiera positivamente en el desarrollo de las dimensiones cognitiva, moral y, por ende, motora. De igual forma, la aparición de la actividad deportiva en el contexto escolar constituyó un proceso que acompañó el cumplimiento de las funciones de la formación moral, de control "racional" de la disciplina y de la conducta. Esto convirtió al deporte desde su inicio en importante instrumento formativo al servicio de los objetivos educativos (MEN, 2000).

En el contexto histórico nacional y a partir de la inferencia de las normas que han regido la educación colombiana, desde la época de la Colonia hasta la Constitución Política de 1991 se pueden vislumbrar diferentes concepciones de la educación física. La primera, y principal, como forma de sometimiento y castigo; integrada a la formación moral, militar e higiene; asignatura obligatoria integrada a la moral, al orden social y a la higiene; como medio de socialización, formación de la personalidad, vigorización de la salud del pueblo. Igualmente, como proceso de formación cívica y social, físico-deportiva y de rendimiento de la juventud; educación física al servicio del deporte; psicomotricidad en preescolar y primaria; como derecho fundamental, servicio público y función social; como un proceso de formación permanente, social y cultural y área fundamental y obligatoria; finalmente, como disciplina pedagógica, práctica social, disciplina del conocimiento y derecho del ser humano (MEN, 2000).

En el marco educativo actual se permite elaborar un concepto de educación física como práctica social, disciplina del conocimiento, disciplina pedagógica 
y como derecho del ser humano que la fundamentan como un proceso de formación permanente, personal, cultural y social a través de la actividad física, recreativa y deportiva para contribuir al desarrollo del ser humano en la multiplicidad de sus dimensiones (MEN, 2000). Los discursos que fundamentan la concepción de educación física son diversos, se han caracterizado por su respuesta y adaptación de contenidos a las demandas sociales, políticas y culturales propias de cada época (Rosa, 1992).

\section{Concepción de cuerpo}

Cuando se habla sobre cuerpo humano es necesario situarse en un punto de partida en torno a él, pues existen varias miradas que pretenden explicarlo o comprenderlo desde su óptica particular (Courtine, Corbin y Vigarello, 2005; Le Breton, 2002). Por ejemplo, si se observa la evolución humana, se puede evidenciar en la filogénesis, en la humanización de la especie, una morfogénesis del cuerpo del Homo sapiens, si se entiende esta evolución de lo corporal como un armazón maleado por la genética. 0 también una sociogénesis, si se tienen en cuenta, además del factor genético, las interacciones sociales, demográficas, cosmogónicas y ecológicas que llevaron a la especie a una narrativa del cuerpo que trasciende el mero estado físico de la materia (Arsuaga y Martínez, 2000).

Esta tendencia de posturas antropológicas dualistas y monistas que se suscitan inconscientemente al citar la morfogénesis y la sociogénesis pueden evidenciarse igualmente en la historia de los cuerpos de la humanidad. Allí la influencia política, económica y de creencias teológicas propendía por cuerpos normativizados o emancipados, según el caso; cuerpos higienizados con fines productivos o, en menor medida, cuerpos libres con la facultad de escoger las mieles hedónicas con sus consecuencias (Le Breton, 1995), o conscientes de la potestad de alcanzar un equilibrio entre las pulsiones y los límites enjuiciadores de las instancias morales (Freud, 2015).

Puede evidenciarse, entonces, que las dos concepciones antropológicas citadas dan origen a una u otra manera de entender el cuerpo humano: físico y simbólico. El cuerpo físico fue sustentado por las ideas antropológicas dualistas de grandes pensadores, como Platón (cuerpo: mundo sensible; alma: mundo de las ideas) y Descartes (cuerpo: sustancia extensa; alma: sustancia pensante); y el cuerpo simbólico se basó en los discursos de las ciencias sociales y la filosofía que aparecieron desde finales del siglo xix y se consolidaron hacia la década de los setenta con autores como: Bertehelot, Durkheim, Fullat, Nietzsche, Freud, Andreu, Fereci, Reich, Dolto, Mauss, Goffman, Bataille, Foucault, Duran, Toro, Gayle, Florenz, Turner, Le Breton, Havaway, Dyaz, Dery, Morave, Ochoa, Butler, López, Planella, Fullat y Villanou.

Los planteamientos de estos últimos pensadores, según Planella (2006), establecen unas concordancias discursivas que posibilitan entender un cuerpo simbólico como construcción social contextualizada; constitutivo de la persona, lo que permite romper con un paradigma dualista para que resurjan el respeto y el cultivo por lo corporal; y eje temático en las investigaciones de las ciencias sociales. Estos estudios sobre el cuerpo le abren la puerta al monismo antropológico, concepción filosófica que sostiene que el hombre está constituido por un solo principio (Meana, 2010).

Sin embargo, a pesar de contar con los aportes de los autores ya referenciados, Planella (2006) es quien establece con mayor claridad la concepción de cuerpo simbólico. Para ello, cita dos acepciones que el idioma alemán ofrece de la palabra cuerpo: körper y Leib. La primera, entiende el cuerpo como objeto pasivo sin posibilidad de ser transformado, como la rex extensa cartesiana, considerado en palabras de Merleau Ponty (1945) como organismo físico. La segunda, se refiere a la dimensión existencial, subjetiva y relacional de la persona, sustancia corpórea en constante transformación, construida culturalmente y decantada por el contexto en que se desenvuelve; en palabras de Ponty (1945), un organismo fenoménico (Sáenz, 2012).

\section{RESULTADOS}

El ejercicio de organización y categorización de la información proporcionada por la muestra del estudio, permitió establecer una matriz de registros de las categorías inductivas (tabla 2).

Tabla 2. Matriz de registros de categorías inductivas

\begin{tabular}{|l|l|c|}
\hline \multicolumn{1}{|c|}{$\begin{array}{c}\text { Categorías } \\
\text { deductivas }\end{array}$} & \multicolumn{1}{|c|}{$\begin{array}{c}\text { Categorías } \\
\text { inductivas }\end{array}$} & $\mathbf{N} .^{\circ}$ registro \\
\hline $\begin{array}{l}\text { Concepción } \\
\text { de educación } \\
\text { física militar }\end{array}$ & $\begin{array}{l}\text { Profesión afín al } \\
\text { ejercicio militar }\end{array}$ & $1-3$ \\
\hline
\end{tabular}




\begin{tabular}{|l|l|c|}
\hline \multicolumn{1}{|c|}{$\begin{array}{c}\text { Categorías } \\
\text { deductivas }\end{array}$} & \multicolumn{1}{|c|}{$\begin{array}{c}\text { Categorías } \\
\text { inductivas }\end{array}$} & N. $^{\circ}$ registro \\
\hline \multirow{4}{*}{$\begin{array}{l}\text { Concepción de } \\
\text { educación física }\end{array}$} & Práctica social & 5 \\
\cline { 2 - 3 } & Derecho humano & 5 \\
\cline { 2 - 3 } & $\begin{array}{l}\text { Disciplina } \\
\text { académico- } \\
\text { pedagógica }\end{array}$ & $6-8$ \\
\cline { 2 - 3 } & $\begin{array}{l}\text { Proceso de } \\
\text { formación }\end{array}$ & 7 \\
\hline \multirow{5}{*}{$\begin{array}{l}\text { Concepción } \\
\text { de cuerpo }\end{array}$} & Cuerpo biológico & 3 \\
\cline { 2 - 3 } & Cuerpo instruido & 4 \\
\cline { 2 - 3 } & $\begin{array}{l}\text { Cuerpo-relación } \\
\text { con los demás y } \\
\text { con el medio }\end{array}$ & 7 \\
\cline { 2 - 3 } & $\begin{array}{l}\text { Cuerpo-sentir- } \\
\text { pensar-actuar-ser }\end{array}$ & 6 \\
\cline { 2 - 3 } & Corporeidad & $5-6$ \\
\hline
\end{tabular}

Fuente: elaboración propia

\section{DISCUSIÓN}

\section{Concepciones de educación física}

\section{Concepción de la educación física como} profesión afín al ejercicio militar

Desde una perspectiva histórica, se sabe que en el nacimiento de la República, en el contexto colombiano, la educación tuvo gran influencia militar. En este periodo se aprecian concepciones de educación física moral militar, que estaban integradas a la filosofía general de la vida, y muy relacionadas con la moral de la época, que requería una nación en proceso de construcción y una formación militar. Cabe recordar que para tal fin, además de incluir la gimnasia militar como parte del pénsum escolar, los estudiantes recibían entrenamiento en prácticas físicas y militares con fusiles y palos. Esta concepción moral-militar de la educación física se mantuvo con algunas variantes durante la reforma educativa realizada por Mariano Ospina Rodríguez en 1844, época en la que se separaron parcialmente las actividades de la escuela de las militares, aunque se reservaron para aquella ciertos principios de orden que son de origen militar (MEN, 2000).

En las siguientes concepciones se logra evidenciar lo enunciado:

"La Educación Física es una profesión complementaria que desde una perspectiva funcional le brinda a los futuros oficiales del ejército (...) y desde la misión normalizadora de las condiciones y paz y seguridad del país". (Registro 1)
“... es absolutamente afín al ejercicio militar y coherente con las actividades diarias de la vida militar". (Registro 3)

\section{Concepción de la educación física como práctica social}

Un docente participante expresa, acerca del concepto de educación física, que "es un proceso de desarrollo del ser humano consigo mismo, con la naturaleza y la cultura, que se expresa en diferentes dimensiones: como práctica social" (Registro 5).

Al considerar que la educación cumple funciones sociales, esta se establece entonces como una actividad intencionada e igualmente reglada por la sociedad. Así como la Constitución definió la educación como un derecho de la persona, la fijó como un servicio público y le asignó sus finalidades. En este sentido, si se asume la educación física como un fin educacional en sí mismo, se puede decir que como componente de la educación también cumple unas funciones asignadas por la misma sociedad. Estas funciones han sido determinadas de acuerdo con las circunstancias y momentos históricos, sociales y culturales. En definitiva, en la sociedad actual asume la responsabilidad de satisfacer necesidades fundamentales de calidad de vida, bienestar y procesos de convivencia. Ha sido pensada para posibilitar el pleno desarrollo de la personalidad de los individuos, fortalecer el respeto por los derechos humanos y promover la tolerancia y otros valores. De esta manera contribuye a reforzar las finalidades propuestas para la educación en general (MEN, 2000).

\section{Concepción de la educación física como derecho humano}

La categoría del concepto de educación física como derecho humano surge de lo expresado por un docente participante, quien considera que la educación física "es un proceso de desarrollo del ser humano consigo mismo, con la naturaleza y la cultura, que se expresa en diferentes dimensiones: (...) como derecho humano" (Registro 5).

En el contexto colombiano, el establecer la educación física como derecho humano implica ubicarla en el marco de la Constitución Política de 1991, a través de la cual se generaron cambios sustanciales muy importantes en todos los ámbitos, incluido el educativo (Constitución Política de Colombia, 1991). Entre los cambios institucionales más importantes que se produjeron, se encuentra la definición de Colombia como un Estado 
social de derecho, fundado en el respeto a la dignidad humana y en los principios y valores de la modernidad. Esta definición tiene origen en la doctrina de los derechos humanos, según la cual todos los hombres y las mujeres tienen unos derechos fundamentales que son inalienables, inherentes y esenciales a la persona (Asamblea General de las Naciones Unidas, 2013).

\section{Concepción de la educación física como disciplina académico-pedagógica:}

"Como lo ha planteado Gruppe, es la pedagogía pues esta se ocupa de la totalidad humana y no de una parte de ella. Siguiendo al autor, la pedagogía se constituye en la categoría integradora de los aportes de las diferentes disciplinas auxiliares que, desde la transposición didáctica, conforman la región y conocimiento propio de la Educación Física, orientado a la educación de esa complejidad que es lo humano". (Registro 6)

“... La educación física es una disciplina académicopedagógica que pretende educar la corporeidad y lograr una comprensión del ser humano, de su propio cuerpo en relación a [sic] la sociedad que le corresponde vivir". (Registro 8)

Siguiendo lo anterior, el valor pedagógico de la educación física y el punto de partida de su rigor académico radican en afirmar que toda acción formativa que parta del movimiento humano es el principal medio educativo de la persona (Bonilla y Bolívar, 1995, p. 35). Así, cuando de manera premeditada, sistemática y declarada, las actividades físicas se convierten en medio de formación de valores, hábitos, habilidades y conocimiento, se está en presencia de la educación física como pedagogía; su finalidad central consiste en la formación de ciudadanos para un tipo de sociedad (Bonilla y Bolívar, 1995, p. 174). De esta manera, la educación física es una

Disciplina pedagógica que asume una tarea de formación personal y social de tal naturaleza que el alcance de sus logros es caracterizado por la visión desde la cual se establezcan relaciones e interrelaciones en distintos campos del desenvolvimiento del ser humano, sus formas de movimiento y expresión, las significaciones de la acción y su sentido. (MEN, 2000, p. 17).

Al respecto, Portela (2001) afirma que la pedagogía es una disciplina que investiga, aplica y experimenta acerca de la enseñanza de un saber específico en un lugar determinado; esto sugiere que la comprensión de la educación física y la pedagogía debe darse en los contextos disciplinar y social-particular. Lo disciplinar como la posibilidad de revisar las prácticas pedagógicas a partir de los discursos de los que nos valemos y desde la interacción con las distintas disciplinas, necesariamente cruzadas, desde las concepciones de cuerpo-movimientocultura. Lo social-particular corresponde a las necesidades, los sueños y las expectativas por satisfacer, desde una mirada en la complejidad de las relaciones humanas (el ser feliz a través del movimiento humano).

Concepción de la educación física

como proceso de formación

... la educación física es un proceso continuo de formación que permite conocerse a sí mismo y como consecuencia desarrollar conductas acerca del cuidado de sí, de las relaciones óptimas con los demás y con el medio. (Registro 7)

El concepto de educación física como proceso de formación implica entenderla como el área de la educación que propende por el desarrollo inteligente del cuerpo, que le permite al sujeto relacionarse con su contexto, independientemente de las condiciones y circunstancias. Así lo expresa la Ley del Deporte y la Educación Física en Colombia:

Entiéndase por educación física la disciplina científica cuyo objeto de estudio es la expresión corporal del hombre y la incidencia del movimiento en el desarrollo integral y en el mejoramiento de la salud y la calidad de vida de los individuos. (MEN, 1995, p. 4).

\section{Concepciones de cuerpo}

La concepción de cuerpo manifestada por los docentes participantes oscila entre las visiones dualista y monista. Dualista: cuerpo biológico, cuerpo instruido. Monista: cuerpo biofísico-psíquico-social, corporeidad, cuerpo sentir-pensar-actuar-ser, cuerpo relación con los demás y con el medio, cuerpo relación con el contexto (religión-filosofía-sociedad-ciencia).

Visiones dualistas del cuerpo

Cuerpo biológico

"... considero que este concepto se relaciona directamente con las capacidades motrices y su desarrollo" (Registro 3). El registro anterior permite inferir la 
concepción de un cuerpo considerado básicamente materia, lo que orienta el interés hacia el desarrollo de las capacidades motrices, es decir, a ser reducido a lo puramente biológico y sustentado en el hecho de considerar el espíritu humano como una superestructura de los fenómenos corporales, en contravía de lo que manifiesta Nietzche (citado por Gruppe, 1976, p. 7): "yo soy total y plenamente cuerpo y nada más, el alma no es más que una palabra para expresar algo que en realidad pertenece al cuerpo".

Cuerpo instruido

“... el militar debe preparar su cuerpo para responder físicamente a la exigencia de la vida militar" (Registro 4). Se observa la marcada inclinación por "instruir" el cuerpo del combatiente, ya que, precisamente, el lema de la Facultad de Educación Física Militar es "Buscaré la disciplina del cuerpo, la superación de mi espíritu" (Escuela Militar de Cadetes, 2017). Se procura conseguir un cuerpo entrenado y saludable (Latham, Anderson, Lee, Bennett, Moseley, Cameron \& Group, 2003).

Visiones monistas del cuerpo

"Cuerpo es la forma en que hacemos presencia en el mundo, con él tenemos las experiencias que nos constituyen como sujetos, establecemos relaciones con los demás y con el medio. Con él, es que recordamos, vivimos y construimos realidad" (Registro 7). El anterior registro presume una mirada del cuerpo desde una postura antropológica monista y permite hacer las siguientes afirmaciones.

\section{Cuerpo-relación con los demás y con el medio}

En la dimensión corporal la naturaleza humana se conjuga de manera dinámica, constante y en muchas situaciones impredecible, con el mundo de la cultura. Así conforma un hecho específico: la experiencia corporal, que se constituye en la complejidad de acciones y relaciones del ser humano con el mundo que dan lugar a las vivencias y la construcción de experiencias eróticas, éticas, estéticas, cognitivas y expresivas que tienen como condiciones el desarrollo de capacidades y competencias para su apropiación, aplicación y transformación.

Cuerpo-sentir-pensar-actuar-ser

Como una concepción de "ser cuerpo", tal como lo expresa Cagigal (1979, p. 74): “el cuerpo también puede ser objeto de reflexión y conciencia, de crítica, de disfrute y de experimentación [...] el hombre seguirá viviendo toda su existencia no solo en el cuerpo, sino con el cuerpo".

Corporeidad

“... Los enfoques contemporáneos reconocen el cuerpo en su naturaleza biológica, física, psíquica y social, en sus características objetivas y subjetivas y en sus relaciones que generan conceptos como corporeidad" (Registro 5). La corporeidad parte de la noción de "estar en el mundo"; es el cuerpo entendido como el ámbito existencial de la cultura (Cordero, 2015).

... La categoría [corporeidad] sintetiza esta realidad existencial de lo humano y exige a la educación física asumirla de manera holística en los procesos formativos. De esta manera se supera el dualismo mente-cuerpo que llevó a dividir lo educativo en áreas "las más importantes" que se ocupan de la mente y otras como la educación física a ocuparse del cuerpo, entendido este como lo biológico-orgánico solamente. (Registro 6)

\section{CONCLUSIONES}

El análisis de las categorías inductivas del estudio evidencia un delimitado espectro con relación a la concepción de educación física militar, pues solo se considera como profesión afín al ejercicio militar. Esto deja de lado categorías como práctica social, derecho humano, disciplina académico-pedagógica, proceso de formación. En cuanto a la concepción de cuerpo la cuestión es similar, se citan únicamente las categorías cuerpo biológico, cuerpo instruido, y se desconocen las categorías cuerpo-relación con los demás y con el medio, cuerpo-sentir-pensar-actuar-ser, corporeidad. Por lo anterior, se podría afirmar que la marcada predominancia de hábitos e ideas castrenses en la cotidianidad del contexto militar promueve una educación física y cuerpos normativizados en busca de la eficiencia bélica y el rendimiento físico. Esto, por un lado, muestra una ventaja, epistemológicamente hablando, al favorecer la delimitación de su objeto de estudio; por el otro, precisa de una más profunda fundamentación que posibilite su metaconocimiento, mayor rigurosidad teleológica y ratificación de la denominación del programa Profesional en Educación Física Militar. 


\section{REFERENCIAS}

Arsuaga, J. L. y Martínez, I. (2000). La especie elegida: la larga marcha de la evolución humana. Madrid: Ediciones de Bolsillo.

Asamblea General de las Naciones Unidas (2013). Declaración Universal de Derechos Humanos. Ginebra: Naciones Unidas. Recuperado de http://www.ohchr.org/ Documents/Publications/UNDRIPManualForNHRIs_ SP.pdf.

Bachelard, G. (1973). Epistemología. Barcelona: Anagrama.

Bonilla, C. y Bolívar, C. (1995). Pedagogía y cultura física. Editorial Kinesis. Armenia.

Bonilla, E. y Rodríguez, P. (1997). Más allá del dilema de los métodos. Santa Fe de Bogotá: Ediciones UniandesNorma.

Cagigal, J. M. (1979). Cultura intelectual y cultura física. F. Hinkelbein y R. C. Muros (eds.). México: Kapelusz.

Camargo, M. (1995). Hacia la construcción de una etnografía del adolescente. Proyecto Atlántida. Santa Fe de Bogotá: upn-ciup-fes.

Centro Nacional de Acreditación. (2012). Informe de evaluación externa con fines de acreditación, Programa de Profesional en Educación Física Militar. Bogotá.

Cerda, H. (1995). Los elementos de la investigación: ¿cómo reconocerlos, diseñarlos y construirlos? (2. ㄹ ed.). Santa Fe de Bogotá: El Búho.

Constitución Política de Colombia. (1991). Bogotá: Leyer.

Cordero, F. O. G. (2015). Del cuerpo a la corporeidad. Educación y Ciudad, 28.

Courtine, J. J., Corbin, A. y Vigarello, G. (2005). Historia del cuerpo. Madrid: Taurus Ediciones.

Ejército Nacional de Colombia. (2016). Principios y valores. Recuperado de https://www.ejercito.mil.co/ index.php?idcategoria $=268892$

Escuela Militar de Cadetes. (2017). Educación Física Militar. Recuperado de http://190.26.215.242/esmic/ index.php/incorporaciones/descargar-recibos/doc_ download/12-35536189

Fernández, R. (2007). Memoria y olvido de Francisco Amorós y de su modelo educativo gimnástico y moral. Ricyde, Revista Internacional de Ciencias del Deporte, 3(6).

Freud, S. (2015). Tres ensayos para una teoría sexual. PARÍs: FV ÉDITIONS.

Gruppe, 0. (1976). Teoría pedagógica de la educación física. Madrid: inef.
Hernández-Sampieri, R.; Fernández-Collado, C. y BaptistaLucio, P. (2010). Metodología de la investigación (5.a ed.). México: McGraw-Hill.

Kuhn, T. (1969). La estructura de las revoluciones científicas. Madrid: Fondo de Cultura Económica.

Latham, N. K., Anderson, C. S., Lee, A., Bennett, D. A., Moseley, A., Cameron, I. D. \& Group, F. T. F. C. (2003). A randomized, controlled trial of quadriceps resistance exercise and vitamin D in frail older people: the Frailty Interventions Trial in Elderly Subjects (fitness). Journal of the American Geriatrics Society, 51(3), 291-299.

Le Breton, D. (1995). Antropología del cuerpo y modernidad/Anthropologie du corps et modernité. París: Nueva Visión.

Le Breton, D. (2002). La sociología del cuerpo. Buenos Aires: Nueva Visión.

Meana, D. I. R. (2010). El cuerpo humano como subjetividad según Edith Stein. En torno al monismo antropológico. Pensamiento. Revista de Investigación e Información Filosófica, 66(249), 833-845.

Merleau-Ponty, M. (1945). Phénoménologie de la perception. Paris: Gallimard.

Ministerio de Educación Nacional. (1995). Ley 181 de enero 18 de 1995 por el cual se dictan disposiciones para el fomento del deporte, la recreación, el aprovechamiento del tiempo libre y la Educación Física y se crea el Sistema Nacional del Deporte. Recuperado de http://www. mineducacion.gov.co/1621/articles-85919_archivo_ pdf.pdf

Ministerio de Educación Nacional. (2000). Lineamientos Curriculares Educación Física, Recreación y Deportes. Bogotá: Magisterio.

Planella, J. (2006). Cuerpo, Cultura y Educación. Bilbao: Desclée de Brouwer.

Portela, H. (2001). La epistemología en la educación física. Manizales: Universidad de Caldas.

Rodríguez, J. M. (2011). Métodos de investigación cualitativa. Revista de Investigación Silogismo, 1(8).

Rosa, M. (1992). Historia de la Educación Física. Cuadernos de Sección. Educación, 5, 27-47.

Sáenz, C. L. (2012). Merleau-Ponty (1908-1961) y Simone de Beauvoir (1908-1986). El cuerpo fenoménico desde el feminismo. Sapere Aude-Revista de Filosofia, 3(6), 182-199.

Tezanos, A. de. (1998). Una etnografía de la etnografía. Aproximaciones metodológicas para la enseñanza del enfoque cualitativo-interpretativo para la investigación social. Santa Fe de Bogotá: Anthropos. 